Agronomía Costarricense 40(2): 77-86. ISSN:0377-9424 / 2016

www.mag.go.cr/rev_agr/index.html www.cia.ucr.ac.cr

\title{
DINÁMICA DE LA SOMBRA DE Cordia alliodora EN SISTEMAS AGROFORESTALES CON CAFÉ EN TOLIMA, COLOMBIA
}

\author{
Hernán Jair Andrade ${ }^{1 / *}$, Milena Andrea Segura** \\ Palabras clave: Área de sombra; incremento de sombra; diseño y manejo; regresión no lineal; oclusión. \\ Keywords: Shade area; shade increment; design and management; non-lineal regression; occlusion.
}

Recibido: 29/03/16

\section{RESUMEN}

Se construyó un modelo de regresión no lineal para explicar la sombra generada por árboles individuales de Cordia alliodora en función de su edad en sistemas agroforestales con café en el Líbano, Tolima, Colombia. Se midió el diámetro del tronco a la altura del pecho, el área de copa y se estimó la oclusión de la copa con fotografía hemisférica de 50 árboles individuales entre 1 y 19 años. Se estudiaron 14 modelos genéricos de regresión no lineal hasta desarrollar el hiperbólico que fue seleccionado por el de mejor ajuste debido a su menor cuadrado medio del error y los Criterios de Información de Akaike y Bayesiano. Se desarrolló una herramienta práctica para los productores de café, con la cual pueden estimar la densidad de $C$. alliodora que suministra el nivel de sombra deseado. Esta herramienta se construyó con base en los estimados de sombra de árboles individuales del modelo de crecimiento y la sombra necesaria para el manejo del cultivo. La copa de $C$. alliodora presentó promedios de oclusión de 0,79 y de área de copa de 60,4 $\mathrm{m}^{2}$.individuo ${ }^{-1}$. El crecimiento de la sombra es mayor en árboles jóvenes que en adultos (5,5 vs 3,0 $\mathrm{m}^{2}$.individuo ${ }^{-1}$.año ${ }^{-1}$ para árboles menores de 5 años y cerca de 20 años, respectivamente). De la misma manera, los árboles de C. alliodora jóvenes presentaron en promedio de $20 \mathrm{~cm}^{2} \mathrm{de}$

1 Autor para correspondencia. Correo electrónico: hjandrade@ut.edu.co

* Universidad del Tolima, Facultad de Ingeniería Agronómica, Colombia.
Aceptado: 01/08/16
** Universidad del Tolima, Facultad de Ingeniería Forestal, Colombia. 
área basal por $\mathrm{m}^{2}$ de sombra en comparación con $62,7 \mathrm{~cm}^{2} \cdot \mathrm{m}^{-2}$ en los mayores. Los resultados de investigación y la herramienta generados en este estudio son claves para el diseño y manejo de la sombra por parte de productores cafeteros, ya que permite definir la densidad de árboles necesarios.

\section{INTRODUCCIÓN}

La caficultura en Colombia, es una de las actividades económicas más importantes ya que ha contribuido con un 8,6 a 11,7\% del PIB agrícola entre el 2000 y 2010 y se estima la participación de unas 560000 familias cafeteras en el 2012 (SIC 2012). Para este estudio en particular, se consideró el Municipio del Líbano ya que es el segundo productor del Tolima, y a su vez es el tercer departamento con mayor producción en el país (FEDERACAFÉ 2016). Como antecedente se identificó que cerca del $50 \%$ de las 920 mil hectáreas de cafetales en Colombia, se cultivan bajo algún tipo de sombrío (Farfán 2014), los cuales podrían aplicar al pago por servicios ecosistémicos o certificaciones de manera que esa condición contribuya al incremento de ingresos de los productores (Segura y Andrade 2012).

Dentro de esos procesos ecositémicos, se consideran los sistemas agroforestales (SAF), que incorporan especies leñosas perennes a sus componentes tradicionales de producción agropecuaria (Somarriba 1992, Nair 1993) que bien diseñados son opciones de manejo sostenible en fincas agropecuarias. Estos sistemas pueden generar bienes y servicios que mejoran los medios de vida rurales (Beer et al. 2003). El manejo de la sombra es probablemente la interacción biofísica más importante en los SAF tropicales, principalmente en sitios sin épocas marcadas de déficit hídrico (Andrade et al. 2008). Por otra $\mathrm{cm}^{2} \cdot \mathrm{m}^{-2}$ in the adults. Research results and the tool generated from this study are key for the design and management of the shade by coffee producers since it allows defining the density of trees needed.

parte, el éxito de sistemas mixtos tal como los agroforestales, dependen del balance entre interacciones negativas y positivas así como la relación cultivo-árboles, principalmente por la competencia de luz, agua y nutrientes (Jose et al. 2000).

En este particular, se ha considerado que la sombra para el cultivo de café genera beneficios productivos y ecológicos, tal como la conservación de biodiversidad, mitigación del cambio climático y regulación microclimática (Perfecto et al. 1996, DaMatta y Rodríguez 2007, Andrade et al. 2014, Häger et al. 2014, Guzmán et al. 2016) y adaptación al cambio climático (Perfecto et al. 2007, Tscharntke et al. 2011, Turbay et al. 2014). Otra razón importante para mantener árboles de sombra en plantaciones como los cafetales, es la producción de frutas o madera, lo cual puede complementar los ingresos de los productores en épocas de precios bajos del café (Beer et al. 1998).

El entendimiento de la dinámica de la sombra es fundamental para su diseño y manejo (Somarriba 2002). El nivel óptimo de sombra para los cultivos es multifactor ya que depende de la tolerancia a sombra y condiciones edáficas y climáticas (Muschler 1999). En Colombia, la FEDECAFÉ recomienda un $30 \%$ de sombra en cafetales, lo cual coincide con la maximización de la fijación de carbono en biomasa en el Líbano, Tolima, Colombia (Andrade et al. 2014). En contraste, la producción de café puede reducirse un $39 \%$ al tenerse bajo una sombra del $60 \%$ de $C$. 
alliodora (Farfán y Urrego 2004). Igualmente la calidad de sombra expresado en términos de área y densidad de copa (oclusión), que requiere de decisiones de manejo bien informadas (Tscharntke et al. 2011).

El objetivo del presente estudio fue evaluar la dinámica de la sombra de árboles individuales de nogal cafetero (Cordia alliodora) en el municipio del Líbano, Tolima, Colombia. Adicionalmente, se presenta una herramienta práctica para la estimación, diseño y manejo de la sombra de esta especie arbórea en los cafetales.

\section{MATERIALES Y MÉTODOS}

El municipio se encuentra a $1565 \mathrm{~m}$ de altitud, $2235 \mathrm{~mm}$ de precipitación anual y $19,1^{\circ} \mathrm{C}$ de temperatura promedio. Los suelos de la zona de estudio son clasificados como Andisoles, con buenas características físicas, alta fertilidad y pendientes entre 25 y $60 \%$ (CIAT 2011). Líbano contiene una alta variedad de sistemas de producción que van desde el café en monocultivo y los SAF con musáceas, caucho (Hevea brasiliensis) y C. alliodora (Andrade et al. 2014).

Se seleccionaron 11 fincas sembradas de café con SAF de $C$. alliodora con una área mínima de 1 ha, y árboles de 1 a 19 años que presentaban diferente nivel de sombra. Dentro de éstos, se escogieron aleatoriamente 50 individuos de la especie en estudio, los cuales eran típicos y representativos de los encontrados en el sistema. La edad de cada individuo fue consultada a los productores. A cada individuo se le midió el diámetro del tronco a la altura del pecho (dap) con cinta diamétrica, 2 diámetros de copa de la proyección vertical de la misma sobre el suelo (líneas verticales del borde de la copa hasta el suelo) y se tomaron 2 fotografías, hacia arriba completamente verticales, a la copa con una cámara digital Canon EOS Rebel XS con un lente de $108^{\circ}$ de apertura. El área de copa se estimó como el círculo cuyo diámetro es el promedio de los medidos.
Se estimó el área basal de cada árbol, a partir del área transversal del tronco a una altura de 1,3 m como un círculo, cuyo diámetro es el dap. Las fotografías de la copa se analizaron con el software Gap Light Analyzer versión 2.0 (GLA; Frazer et al. 1999), con la estimación de la oclusión, la cual se define como la fracción de suelo en el cual la copa intercepta los rayos solares (Bellow y Nair 2003). El área de análisis de cada fotografía incluyó solo la copa de ese individuo, sin tener traslapes con otras copas. El área de sombra por árbol se estimó como el producto del área de copa y el factor de oclusión.

Se probaron 14 modelos de regresión no lineal para encontrar la ecuación de mejor ajuste que describiera el crecimiento de la sombra de los árboles en función de su edad. En la selección del modelo de mejor ajuste se consideró aquel que tuviera el menor valor del cuadrado medio del error (CME) y los Criterios de Información de Akaike (AIC) y Bayesiano (BIC). De la misma forma, se graficaron los 3 mejores modelos con base en los criterios mencionados y se observó su lógica biológica y se realizó el ajuste de los datos. Se estimó el indicador $\mathrm{Ab} /$ As, resultado del cociente entre área basal y área de sombra, que indica el potencial volumen de madera que produce por unidad de sombra que proporciona al cultivo. Entre mayor sea este indicador mayor es el potencial de producción de madera en función del área de sombra generada al estrato inferior del sistema de producción. Todos los análisis estadísticos fueron desarrollados en Infostat.

Adicionalmente, se desarrolló una herramienta básica y de fácil empleo que facilita estimar la densidad de árboles de $C$. alliodora en función de su edad y la sombra deseada para el cafetal. La herramienta fue diseñada con el modelo de mejor ajuste generado, que estima la sombra en función de la edad de los árboles. Esto permite que los productores puedan tomar decisiones sobre el manejo de la sombra con esta especie arbórea en sus cafetales. 


\section{RESULTADOS Y DISCUSIÓN}

Se encontró que los árboles tenían un área de copa promedio de $60,4 \mathrm{~m}^{2}\left(4,8-205,4 \mathrm{~m}^{2}\right)$ con un factor de oclusión de la copa de 0,79. Es decir, la copa obstruye el $79 \%$ de la radiación incidente sobre la copa, para dejar un $21 \%$ de claros. La oclusión encontrada en este estudio coindice con lo manifestado por productores de café en Costa Rica, quienes afirman que la copa de C. alliodora es abierta (Cerdán et al. 2012), lo cual se confirma con la estimación de su coeficiente de extinción de radiación solar de 0,63 (Castaño et al. 2013). En promedio, se encontró que cada árbol incrementa anualmente su área de sombra en 4,1 $\mathrm{m}^{2}$. Esta información es clave para el manejo de la sombra ofertada por esta especie.
En el Cuadro 1 se detallan los modelos de regresión no lineal que fueron probados para encontrar el de mejor ajuste. El modelo hiperbólico resultó ser el que mejor se ajustó a los datos colectados, ya que presentó los menores valores de cuadrado medio del error y de los Criterios de Información de Akaike y Bayesiano (Cuadro 1; Figura 1). De la misma forma, dentro del rango de edades estudiado (1 a 19 años), la curva trazada por este modelo sigue una lógica biológica. Este modelo describe la dinámica del área de sombra de árboles individuales, y muestra que el mayor incremento en la sombra se alcanza en árboles menores de 5 años $\left(5,1\right.$ a $5,8 \mathrm{~m}^{2}$.individuo ${ }^{-1}$. $\mathrm{año}^{-1}$; Figura 1). Este valor se disminuye conforme el árbol crece, hasta llegar cerca de 3,0 $\mathrm{m}^{2}$.individuo ${ }^{-1}$.año ${ }^{-1}$ en árboles de 20 años. 
ANDRADE Y SEGURA: Sombras de Cordia alliodora en sistemas agroforestales

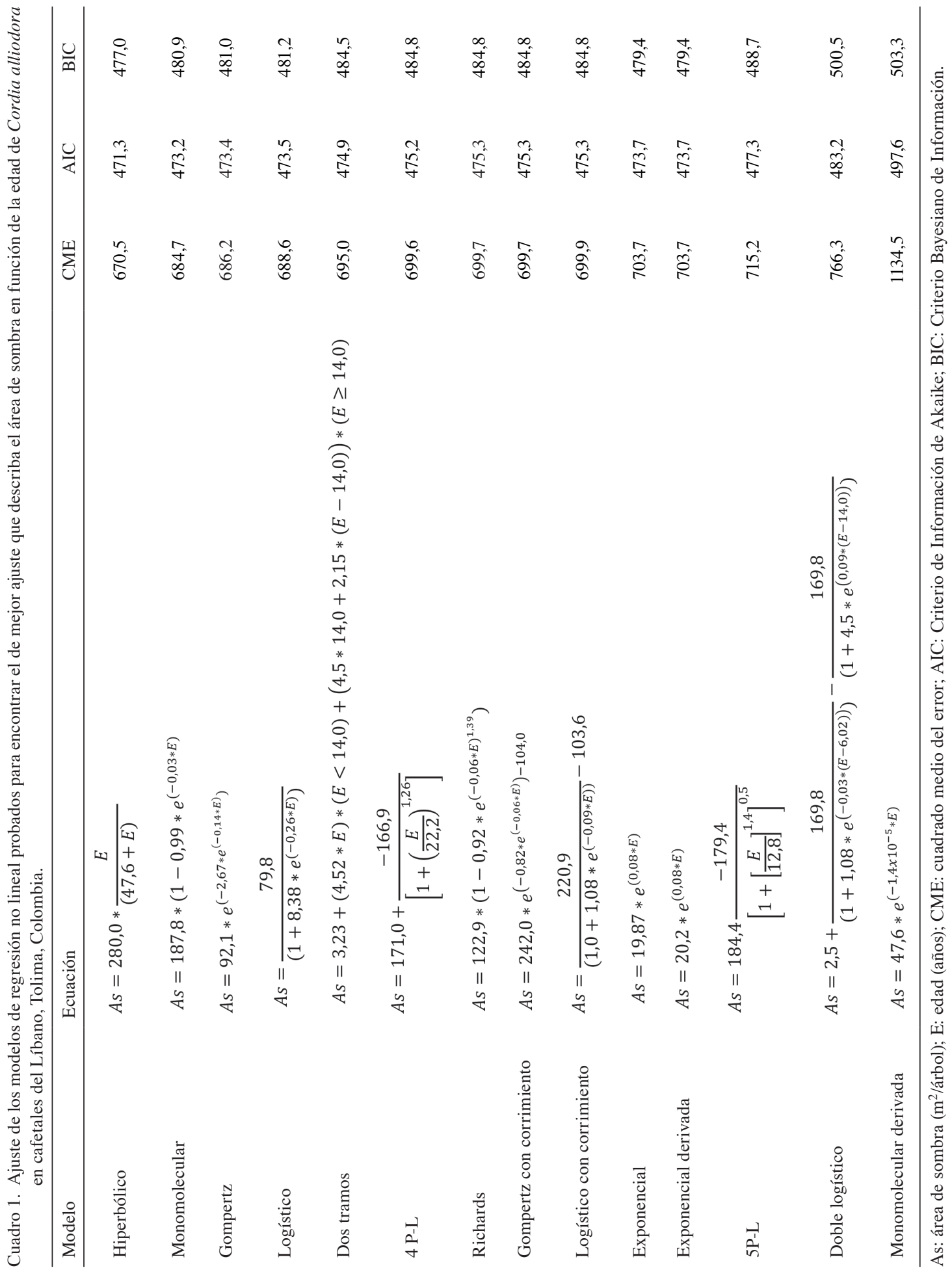




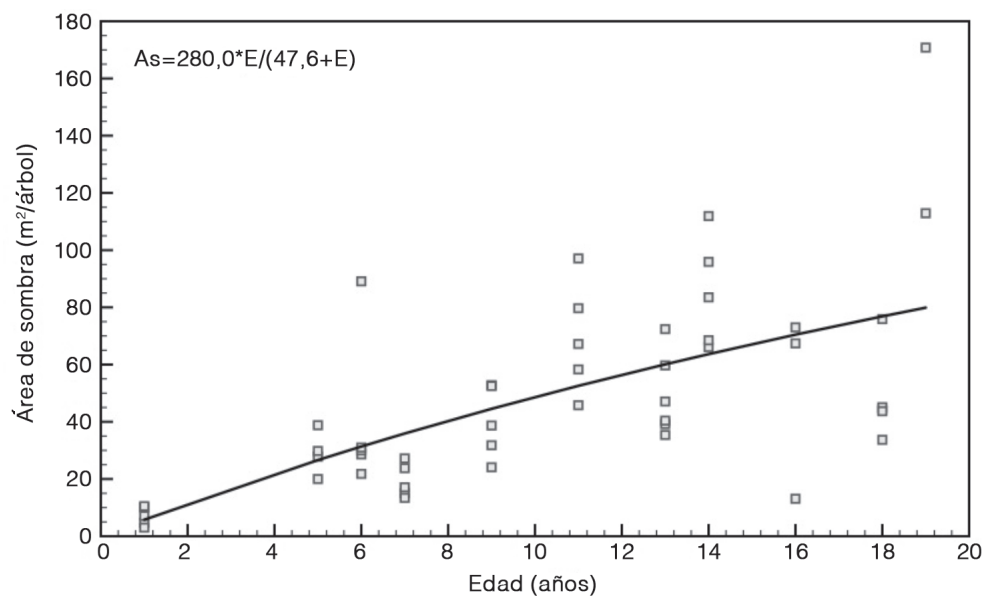

Fig. 1. Comportamiento del área de sombra de árboles individuales de C.alliodora en función de la edad en cafetales del Líbano, Tolima, Colombia.

As: área de sombra; E: edad.

En general, el comportamiento del área basal en función del área de sombra, dado por la relación $\mathrm{Ab} / \mathrm{As}$, es constante en los árboles jóvenes ( $\leq 14$ años): $20 \mathrm{~cm}^{2} / \mathrm{m}^{2}$ (Figura 2). En contraste, los árboles mayores tienden a tener relaciones $\mathrm{Ab} / \mathrm{As}$ más variables: $62,7 \pm 15,3 \mathrm{~cm}^{2} /$ $\mathrm{m}^{2}$ (Figura 2). Esta tendencia general coincide con los planteamientos de Hemery et al. (2005), quienes afirman que la relación entre el diámetro de copa y el del tronco son altos en árboles jóvenes y se reduce al aumentar el tamaño de tronco. En contraste, en plantaciones de $C$. alliodora en Costa Rica no se encontró un efecto de la densidad (> 100 árboles.ha ${ }^{-1}$ ) en la relación entre el diámetro de copa y dap pero sí con la edad (Hummel 2000), similares supuestos fueron empleados por Cañadas et al. (2012).

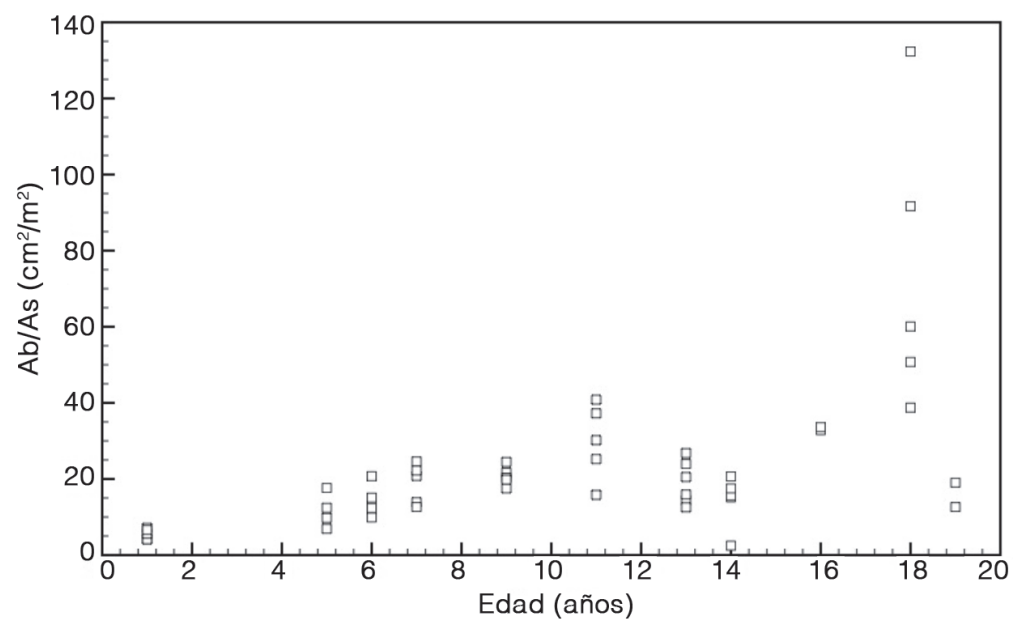

Fig. 2. Dinámica del indicador Ab/As en árboles individuales de C. alliodora en función de la edad en cafetales del Líbano, Tolima, Colombia. 
Los resultados de este estudio indican que para el mismo nivel de sombra, es preferible tener menos árboles de tamaño grande (16 o más años) que árboles pequeños. Con los árboles grandes se puede obtener una mayor área basal al mismo nivel de sombra, lo que implica un mayor potencial de volumen de madera y almacenamiento de carbono en biomasa. Andrade et al. (2012) reportan valores similares a los encontrados en este estudio para las especies maderables en bosques secundarios del Caquetá, Colombia: Capirona sp. Vitex sp. Sapium glandulosum, Cecropia putumayonis, Grias neuberthii y Xanthosoma sagittifolium con un Ab/As de 81, 79, 77, 76, 69 y 69 $\mathrm{cm}^{2} / \mathrm{m}^{2}$, respectivamente.

Se observa una alta variabilidad del indicador Ab/As en aquellos árboles con 18 años (Figura 2). Esta diferencia en la tendencia para esta edad puede obedecer a que estos árboles se encontraban en un SAF de alta densidad arbórea, lo cual reduce el área de copa por competencia de espacio con los árboles vecinos (Seidel et al. 2016). Verman et al. (2014) argumentan que las relaciones entre las dimensiones de la copa y del tronco se afectan en relación con la densidad arbórea. Sin embargo, esto resulta contradictorio con lo manifestado por Hummel (2000), quien afirma que la relación entre los diámetros de copa y del tronco no se afectan por la densidad arbórea.

La Figura 3 es el elemento central de la herramienta para el diseño y manejo de la sombra de $C$. alliodora en cafetales del Líbano. Dicha herramienta permite estimar la densidad de los árboles de esta especie en una edad específica. Cabe señalar que el primer paso para su utilización se sustenta en determinar el área de sombra que se planea ofrecer al cafetal; previamente se deben conocer las características del sitio, el material genético y estado fenológico de los cafetos (Somarriba 2002). Posteriormente, se busca la edad de los árboles en el eje X y se recorre la vertical hasta encontrar la sombra en las curvas, la cual arroja en el eje de las Y la densidad necesaria de $C$. Alliodora, es decir, debe encontrarse la curva en la cual, la edad y la sombra necesaria se interceptan.

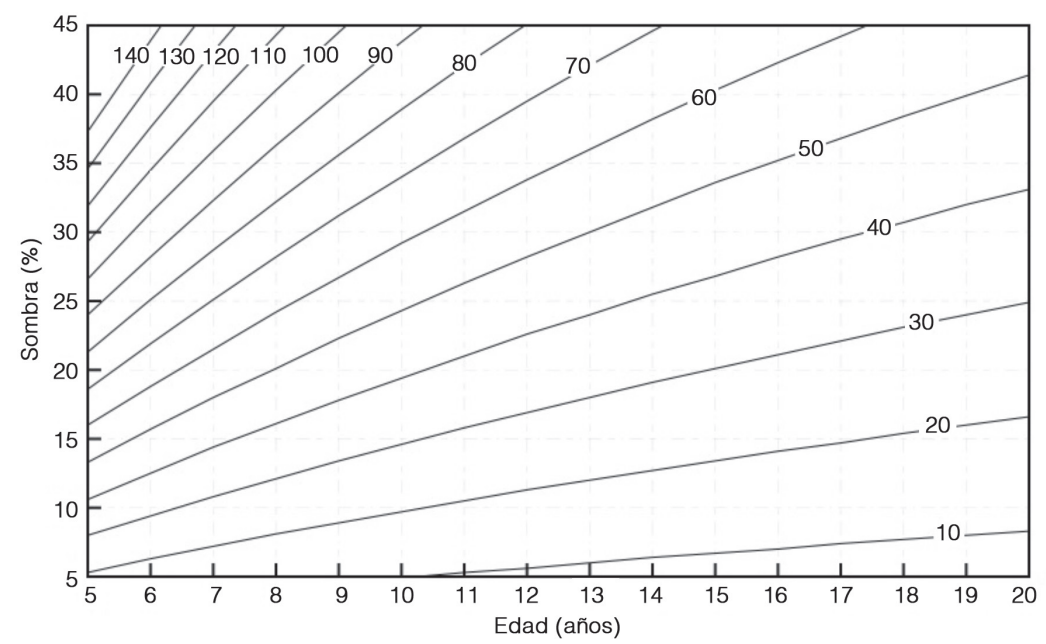

Fig. 3. Herramienta para la estimación de la densidad de árboles de C. alliodora (individuos.ha ${ }^{-1}$ ), demarcados con las curvas en el cuerpo de la figura, de cada edad necesarios para lograr cierto nivel de sombra en cafetales del Líbano, Tolima, Colombia.

Las curvas de 10 a 120 se refieren a la densidad de árboles de cada edad necesarios para alcanzar el nivel de sombra. 
Por ejemplo, si un productor desea suministrar a un cafetal una sombra del $25 \%$ con árboles de 12 años, entonces tendría que tener cerca de 42 árboles.ha $^{-1}$ (Figura 3), en cambio otro productor, en un sitio marginal bajo, por ejemplo necesita $35 \%$ de sombra con árboles de 15 años, en este caso, se establecerían 54 árboles. ha $^{-1}$ (Figura 3). Cabe señalar que esta herramienta en su forma actual, funciona para SAF con rodales de $C$. alliodora coetáneos, es decir, de la misma edad.

Diversos investigadores han estudiado la sombra de $C$. alliodora en cafetales; Andrade et al. (2014) han encontrado un $40 \%$ de sombra en el Líbano, Tolima, Colombia. Bellow y Nair (2003) encontraron resultados idénticos con árboles de 3 a 9 años en Costa Rica.

Righi et al. (2008) afirman que las plantas de café toleran una reducción de la radiación del $50 \%$ sin una caída en el crecimiento e índice de área foliar.

Varios autores han encontrado que la sombra de $C$. alliodora reduce entre 22 y $50 \%$ la producción de café (Glover 1981, Beer et al. 1985, Detlefsen 1988, Farfán y Urrego 2004). En contraste, Soto-Pinto et al. (2000) no encontraron efecto de la sombra en la producción de café en Chiapas, México; mientras que Beer et al. (1985) detectaron que la producción de café bajo nogal (278 árboles.ha ${ }^{-1}$ ) se redujo $28 \%$ respecto a bajo sombra de Erythrina sp. (555 árboles.ha- ${ }^{-1}$ ). Staver et al. (2001) afirman que la producción de café se maximiza con sombras entre 35 y $65 \%$. A pesar de estos hallazgos, muchos productores consideran que la sombra que genera $C$. alliodora es alta y difícil de manejar (Cerdán et al. 2012).

Además de encontrar una diversidad de sombra de $C$. alliodora en cafetales, se han reportado diversos efectos de ésta en el desarrollo y producción de café mientras que el efecto sobre la calidad no es tan claro pues resultados contrastantes se observan según las condiciones del cafetal y del sitio (Bosselmann et al. 2009).

\section{CONCLUSIONES}

La copa de los árboles de $C$. alliodora presentó una oclusión del 0,79 , lo cual indica que el sol deja pasar el $21 \%$ de la radiación al estrato herbáceo. Esta información es clave para el diseño y manejo de la sombra en sistemas forestales $\mathrm{y}$ agroforestales que incluyan esta especie. El modelo de regresión no lineal hiperbólico fue el que mejor ajustó los datos recabados, y además explica de mejor manera, la dinámica de la sombra de esta especie a lo largo de su ciclo de vida.

Se plantea una relación entre el área basal y el área de sombra de los árboles de esta especie, la cual indica el volumen potencial de madera que puede producir un árbol por unidad de área de sombra que proyecta al estrato bajo. Los árboles grandes parecen ser más eficientes por su mayor relación, es decir, producirían más volumen de madera al ofrecer la misma sombra. Finalmente, este estudio desarrolló una herramienta simple y de fácil utilización dirigida a productores cafeteros del área de estudio.

\section{AGRADECIMIENTOS}

Los autores desean expresar su agradecimiento al Comité Central de Investigaciones de la Universidad del Tolima por financiar el Proyecto de Investigación $\mathrm{N}^{\circ}$. 260210, en el marco del cual se desarrolló el presente estudio. A la Federación Nacional de Cafeteros, Sede Líbano, Tolima y principalmente a los caficultores de la zona de estudio. Se expresan los más sinceros agradecimientos a Laura Melo y Daniel Machado, quienes apoyaron en la colecta parcial de datos en campo.

\section{LITERATURA CITADA}

Andrade,HJ; Brook, R; Ibrahim, M. 2008. Growth, production and carbon sequestration of silvopastoral systems with native timber species in the dry lowlands of Costa Rica. Plant and Soil 308(1/2):11-22.

Andrade, HJ; Marín, LM; Pachón, DP. 2014. Fijación de carbono y porcentaje de sombra en sistemas de 
producción de café (Coffea arabica L.) en el Líbano, Tolima, Colombia. Bioagro 26(2):127-132.

Andrade,HJ; Orjuela,JA; Díaz,AI.2012.Especies regeneradas naturalmente con potencialidad de uso como árboles dispersos en pasturas en el departamento del Caquetá, Colombia. Memoria. Congreso Latinoamericano de Sistemas Agroforestales para la Producción Pecuaria Sostenible (4, 2012, Belem do Pará, Brazil) $(8-10,11$, 2012, Belem do Pará, Brasil). p. 607-611.

Andrade, HJ; Segura, MA; Canal, DS; Feria, M; Alvarado, JJ; Marín, LM; Pachón, D; Gómez, MJ. 2014. The carbon footprint of coffee productive chains in Tolima, Colombia. In Oelberman, M (ed.). Sustainable Agroecosystems in Climate Change Mitigation. Wageningen, The Netherlands, Academic Publishers. p. 272.

Beer, J; Harvey, C; Ibrahim, M; Harmand, JM; Somarriba, E; Jiménez, F. 2003. Servicios ambientales de los sistemas agroforestales. Agroforestería en las Américas 10 (37/38):80-87.

Beer, J; Muschler, R; Kass, D; Somarriba, E. 1998. Shade management in coffee and cacao plantations. Agroforestry Systems 38:139-164.

Beer, JW; Fassbender, HW; Heuveldop, J. 1985. Avances en la investigación forestal. San José, Costa Rica. CATIE. Serie Técnica. Informe Técnico $\mathrm{N}^{\circ} 147.451 \mathrm{p}$.

Bellow, JG; Nair, PKR. 2003. Comparing common methods for assessing understory light availability in shadedperennial agroforestry systems. Agricultural and Forest Meteorology 114:197-211.

Bosselmann, AS; Dons, K; Oberthur, T; Olsen, CS; Ræbild, A; Usma, H. 2009. The influence of shade trees on coffee quality in small holder coffee agroforestry systems in Southern Colombia. Agriculture, Ecosystems \& Environment 129(1-3):253-260.

Cañadas, A; Korol, M; Zambrano, C; Camacho, B. 2012. Modelos descriptivos de corona de laurel (Cordia alliodora) bajo sistemas agroforestales en el Bosque Protector Sumaco, Ecuador. Avances 4(2):B31-B38.

Castaño, AM; Chica, HA; Obando, D; Riaño, NM. 2013. Arquitectura del dosel e interceptación de la radiación solar por tres especies forestales nativas en Colombia. Cenicafé 64(1):19-30.

Cerdán, CR; Rebolledo, MC; Soto, G; Rapidel, B; Sinclair, FL. 2012. Local knowledge of impacts of tree cover on ecosystem services in smallholder coffee production systems. Agricultural Systems 110:119-130.

CIAT (Centro Internacional de Agricultura Tropical, Colombia). 2011. Descripción ecotopo Huila Tolima. Cali, Colombia. Consultado 26 jul. 2012. Disponible en ftp://ftp.ciat.cgiar.org/.../descripcion ecotopos\% 20huila-tolima.doc

Damatta, F; Rodríguez, N. 2007. Producción sostenible de cafetales en sistemas agroforestales del Neotrópico: una visión agronómica y ecofisiológica. Agronomía Colombiana 25(1):113-123.

Detlefsen, RG. 1988. Evaluación del rendimiento de Coffea arabica cv. Caturra bajo diferentes densidades de Cordia alliodora y Erythrina poeppigiana plantados en un diseño sistemático de espaciamientos. Tesis M.Sc. Turrialba, Costa Rica, IICA-CATIE. 121 p.

Farfán, F; Urrego, JB. 2004. Comportamiento de las especies forestales Cordia alliodora, Pinus oocarpa y Eucalyptus grandis como sombrío e influencia en la productividad del café. Cenicafé 55(4):317-329.

Farfán, F. 2014. Agroforestería y sistemas agroforestales con café. Manizales, Caldas, Colombia, Federación Nacional de Cafeteros - Cenicafé. 342 p.

FEDERACAFÉ (Federación Nacional de Cafeteros de Colombia). 2016. Tolima café de alta calidad. Bogotá, Colombia. Consultado 28 mar. 2016. Disponible en http://tolima.federaciondecafeteros.org/

Frazer, GW; Canham, CD; Lertzman, KP. 1999. Gap Light Analyzer (GLA) Version 2.0. Burnaby, British Columbia, USA - Millbrook, New York, USA. Simon Fraser University - Institute of Ecosystem Studies. 36 p.

Glover, N. 1981. Coffee yields in a plantation of Coffea arabica var. Caturra shaded by Erythrina poeppigiana with and without Cordia alliodora. Turrialba, Costa Rica. CATIE. Serie Técnica. Informe Técnico N 17. 24 p.

Guzmán, A; Link, A; Castillo, JA; Botero, JE. 2016. Agroecosystems and primate conservation: Shade coffee as potential habitat for the conservation of Andean night monkeys in the northern Andes. Agriculture, Ecosystems \& Environment 215(1):57-67.

Häger, A; Fernández, M; Stuhlmacher, M; Acuña, R; Contreras, A. 2014. Effects of management and landscape composition on the diversity and structure of tree species assemblages in coffee agroforests. Agriculture, Ecosystems and Environment 199:43-51.

Hemery, GE; Savill, PS; Pryor, SN. 2005. Applications of the crown diameter-stem diameter relationship for different species of broadleaved trees. Forest Ecology and Management 215:285-294.

Hummel, S. 2000. Height, diameter and crown dimensions of Cordia alliodora associated with tree density. Forest Ecology and Manegement 127:31-40.

Jose, S; Gillespie, AR; Seifert, JR; Biehle, DJ. 2000. Defining competition vectors in a temperate alley cropping system in the midwestern USA. 2. Competition for water. Agroforestry Systems 48:41-59.

Muschler, R. 1999. Árboles en cafetales. Turrialba, Costa Rica. CATIE. Colección Módulos de Enseñanza Agroforestal N ${ }^{\circ}$. 5. 137 p. 
Nair, PKR. 1993. Agroforestry systems in the tropics. 2ed. Dordrecht, the Netherlands. Kluwer Academic Publishers. 664 p.

Perfecto, I; Armbrecht, I; Philpott, SM; Soto-Pinto, L; Dietsch, TM. 2007. Shaded coffee and the stability of rainforest margins in northern Latin America. In Tscharntke, T; Leuschner, C; Zeller, M; Guhadja, E; Bidin, A (eds.). The Stability of Tropical Rainforest Margins, Linking Ecological, Economic and Social Constraints of Land Use and Conservation. Berlin, Germany. Environmental Science Series, Springer Verlag. p. 227-264.

Perfecto, I; Rice, R; Greenberg, R; Van Der Voort, M. 1996. Shade coffee: a disappearing refuge for biodiversity. Bioscience 46(8):598-608.

Righi, C; Lunz, A; Bernardes, M; Pereira, C; Dourado, D; Favarin, J. 2008. Radiation availability in agroforestry system of coffee and rubber trees. In Advances in agroforestry; toward agro- forestry design: an ecological approach. Springer. p. 249-327.

Segura, MA; Andrade, HJ. 2012. Huella de carbono en cadenas productivas de café (Coffea arabica L.) con diferentes estándares de certificación en Costa Rica. Luna Azul 35:60-77.

Seidel, D; Ruzicka, KJ; Puettmann, K. 2016. Canopy gaps affect the shape of Douglas-fir crowns in the western Cascades, Oregon. Forest Ecology and Management 363:31-38.

SIC (Superintendencia de Industria y Comercio, Colombia). 2012. Estudio sobre el sector del café en Colombia. Estudios de Mercado No. 5. Bogotá, DC, Colombia. Consultado 1 mar. 2016. Disponible en http:// www.sic.gov.co/recursos_user/documentos/ promocion_competencia/Estudios_Economicos/
Estudios_Economicos/Estudios_Mercado/ EstudiosectorialCafe.pdf

Somarriba, E. 1992. Revisiting the past: an essay on agroforestry definition. Agroforestry Systems 19(3):233-240.

Somarriba, E. 2002. Estimación visual de la sombra en cacaotales y cafetales. Agroforestería en las Américas 9(35-36):86-94.

Soto-Pinto, L; Perfecto, I; Castillo-Hernandez, J; CaballeroNieto, J. 2000. Shade effect on coffee production at the northern Tzeltal zone of the state of Chiapas, Mexico. Agriculture, Ecosystems \& Environment 80(1/2):61-69.

Staver, C; Guharay, F; Monterroso, D; Muschler, RG. 2001. Designing pest-suppressive multistrata perennial crop systems: shade-grown coffee in Central America. Agroforestry Systems 53(2):151-170.

Tscharntke, T; Clough, Y; Bhagwat, SA; Buchori, D; Faust, H; Hertel, D; Holscher, D; Juhrbandt, J; Kessler, M; Perfecto, I; Scherber, C; Schroth, G; Veldkamp, E; Wanger, TC. 2011. Multifunctional shade-tree management in tropical agroforestry landscapes - a review. Journal of Applied Ecology 48:619-629.

Turbay, S; Nates, B; Jaramillo, F; Vélez, JJ; Ocampo, OL. 2014. Adaptación a la variabilidad climática entre los caficultores de las cuencas de los ríos Porce y Chinchiná, Colombia. Investigaciones Geográficas, Boletín del Instituto de Geografía 85:95-112.

Verma, NK; Lamb, DW; Reid, N; Wilson, B. 2014. An allometric model for estimating DBH of isolated and clustered Eucalyptus trees from measurements of crown projection area. Forest Ecology and Management 326:125-132. 